J.S. Saczynski, PhD

A. Beiser, $\mathrm{PhD}$

S. Seshadri, MD

S. Auerbach, MD

P.A. Wolf, MD

R. Au, $\mathrm{PhD}$

Address correspondence and reprint requests to Dr. Jane S. Saczynski, Division of Geriatric Medicine, Department of Medicine, 377 Plantation St., Suite 315, Worcester, MA 01605 Jane.saczynski@umassmed.edu

Editorial, page 12

See also pages 21 and 27

\section{Depressive symptoms and risk of dementia}

\author{
The Framingham Heart Study
}

(]

\section{ABSTRACT}

Objectives: Depression may be associated with an increased risk for dementia, although results from population-based samples have been inconsistent. We examined the association between depressive symptoms and incident dementia over a 17-year follow-up period.

Methods: In 949 Framingham original cohort participants (63.6\% women, mean age $=79$ ), depressive symptoms were assessed at baseline (1990-1994) using the 60-point Center for Epidemiologic Studies Depression Scale (CES-D). A cutpoint of $\geq 16$ was used to define depression, which was present in $13.2 \%$ of the sample. Cox proportional hazards models adjusting for age, sex, education, homocysteine, and APOE $\epsilon 4$ examined the association between baseline depressive symptoms and the risk of dementia and Alzheimer disease (AD).

Results: During the 17-year follow-up period, 164 participants developed dementia; 136 of these cases were AD. A total of $21.6 \%$ of participants who were depressed at baseline developed dementia compared with $16.6 \%$ of those who were not depressed. Depressed participants (CES-D $\geq 16$ ) had more than a 50\% increased risk for dementia (hazard ratio [HR] 1.72, 95\% confidence interval [Cl] 1.04-2.84, $p=0.035)$ and AD (HR 1.76, 95\% Cl 1.03-3.01, $p=0.039$ ). Results were similar when we included subjects taking antidepressant medications as depressed. For each 10-point increase on the CES-D, there was significant increase in the risk of dementia (HR 1.46,95\% Cl 1.18-1.79, p < 0.001) and AD (HR 1.39, 95\% Cl 1.11-1.75, $p=0.005)$. Results were similar when we excluded persons with possible mild cognitive impairment.

Conclusions: Depression is associated with an increased risk of dementia and AD in older men and women over 17 years of follow-up. Neurology ${ }^{\circledR}$ 2010;75:35-41

\section{GLOSSARY}

$\mathbf{A D}=$ Alzheimer disease $; \mathbf{C D R}=$ Clinical Dementia Rating; $\mathbf{C E S}-\mathbf{D}=$ Center for Epidemiologic Studies Depression Scale; $\mathbf{C l}=$ confidence interval; $\mathbf{D S M}-\mathbf{I V}=$ Diagnostic and Statistical Manual of Mental Disorders, 4th edition; $\mathbf{H R}=$ hazard ratio; $\mathbf{M C l}=$ mild cognitive impairment; MMSE = Mini-Mental State Examination.

Depression is associated with cognitive impairment in older adults ${ }^{1}$ and a 2001 metaanalysis of case-control and prospective studies of the association between depression and dementia concluded that history of depression approximately doubled the risk for dementia. ${ }^{2}$ Indeed, there is evidence from clinical case-control studies for an association between a baseline history of depression or depressive symptoms and the risk of dementia 10 or more years later. ${ }^{3-5}$ Findings from longitudinal epidemiologic studies, however, are less consistent, with some studies finding that depressive symptomatology was a risk factor for dementia 3-7 years later, ${ }^{6-8}$ and others observing no association, ${ }^{9-11}$ or an association in only selected subgroups. ${ }^{12-14}$ A number of factors may contribute to the inconsistency of findings from longitudinal studies including the length of follow-up, age of study participants, methods of dementia diagnosis and depression screening, and whether or not potentially confounding variables were controlled.

From the Department of Medicine and Meyers Primary Care Institute (J.S.S.), University of Massachusetts Medical School, Worcester; Department of Biostatistics (A.B.), Boston University School of Public Health, Boston; and Department of Neurology (A.B., S.S., S.A., P.A.W., R.A.), Boston University School of Medicine, Boston, MA.

Study funding: Funding information is provided at the end of the article.

Disclosure: Author disclosures are provided at the end of the article. 
This study examines the association between depressive symptoms at baseline and risk of incident dementia over a 17-year follow-up period in participants in the Framingham Heart Study.

METHODS Study sample. The Framingham Heart Study is a longitudinal population-based cohort study of 5,209 subjects ( $55 \%$ women) established in 1948. Since the study's inception, surviving cohort members have undergone regular biennial examinations. A dementia-free inception cohort was established in 1976-1978 when 3,183 subjects (1,361 men, 1,822 women, mean age $71 \pm 8$ years, range 55 to 88 years) were identified as cognitively intact based on performance on a standardized neuropsychological battery in 2,123 subjects, ${ }^{15}$ or on documented normal cognitive status at subsequent examinations and record review.

In 1990, at the start of the 22nd biennial examination cycle, 1,753 subjects from the original cohort were still alive. Of these, $1,166(67 \%)$ attended the 22nd biennial examination and among them, 949 (81\%; 604 woman, 345 men) were dementiafree and were assessed for depressive symptoms. These participants were followed for up to 17 years (average follow-up 8 years) for incident dementia and served as the study population for this analysis.

Standard protocol approvals, registrations, and participant consents. Data were obtained under a protocol approved by the Human Subjects Institutional Review Board of the Boston University School of Medicine. Written informed consent was obtained from all participants.

Depressive symptoms. Depressive symptoms were evaluated using the Center for Epidemiologic Studies Depression Scale (CES-D), ${ }^{16}$ a 20 -item scale consisting of 4 factors: depressive affect, somatic complaints, positive affect, and interpersonal relations. Scores on the CES-D range from 0 to 60 , with higher scores reflecting greater depressive symptoms. Based on the CES-D guidelines, a score of $\geq 16$ was used to indicate high depressive symptomatology. ${ }^{16}$

Treatment for depression with antidepressant medications was documented at the baseline examination and included treatment with selective serotonin reuptake inhibitors, monoamine oxidase inhibitors, tricyclic agents, modified cyclics, and other miscellaneous medications classified as antidepressants.

Incident dementia case ascertainment. Dementia case ascertainment was conducted in a 2-step process which has been described previously. ${ }^{17}$ In brief, participants are first screened using the Mini-Mental State Examination (MMSE), which was administered to all cohort members at each biennial examination beginning at examination cycle 17 (1981). Potential cases were flagged for further follow-up evaluation if their MMSE score fell below age- and education-adjusted cutoffs, fell 3 points from the immediate preceding examination, or fell 5 points across all examinations. Additional information was also used to supplement MMSE scores in flagging possible cases of dementia for further follow-up. This information included findings from the participant's primary care physician or from medical records, findings from ancillary neurologic studies (e.g., the Precursors of Stroke Incidence and Prognosis Study), observations from clinic staff or the examining physician at the regular biennial evaluations, self or family report of change in cognitive status during regular telephone health status updates, or concern regarding cognitive sta- tus expressed by the participant or accompanying family members during any point of contact with Framingham staff. Any of these sources could trigger in-depth follow-up evaluation.

Participants with possible dementia underwent a neurologic examination and 90-minute neuropsychological test battery. Participants with signs of cognitive impairment were reexamined approximately every $1-2$ years to document progression to moderate or severe dementia. All participants classified as having possible or probable dementia were reviewed by a panel consisting of at least 1 neurologist and a neuropsychologist. Participants identified as having dementia satisfied the criteria of the DSM-IV ${ }^{18}$ had dementia severity equivalent to at least 1.0 on the Clinical Dementia Rating $(\mathrm{CDR})^{19}$ scale, and exhibited symptoms of dementia for at least 6 months. Participants identified as having Alzheimer disease $(\mathrm{AD})$ met the criteria of the National Institute of Neurological and Communicative Disorders and Stroke and the Alzheimer's Disease and Related Disorders Association for definite, probable, or possible $\mathrm{AD} .^{20}$

Assessment of control variables. Demographic factors (age, sex, and education), alcohol use (dichotomized into none/any), and current smoking status (smoker, nonsmoker) were assessed at baseline via questionnaire. History of stroke, diabetes, cardiovascular disease, and hypertension was assessed from clinical records. Homocysteine levels were determined with the use of high-performance liquid chromatography with fluorometric detection. ${ }^{21}$ The coefficient of variation for this assay was $9 \% .{ }^{22}$ The presence of particular alleles of apolipoprotein E genotype was determined by means of isoelectric focusing of the plasma and confirmed by DNA genotyping. ${ }^{23,24}$ Participants with an APOE $\varepsilon 4$ allele $(\varepsilon 2 / \varepsilon 4, \varepsilon 3 / \varepsilon 4$, or $\varepsilon 4 / \varepsilon 4$ genotype) were compared to those without an $A P O E \varepsilon 4$ allele. Waist-hip ratio was calculated from measurements taken at the baseline clinical examination.

Statistical analyses. Each participant contributed up to 17 years of follow-up from the baseline examination (cycle 22, 1990-1994) to the development of dementia or censoring (at death, last evaluation, or year 2008). We considered 2 definitions for clinical depression. The first was based on the CES-D score alone and used 16 as the cutpoint for depression $(\geq 16=$ depressed). The second included participants who were taking antidepressant medications in the group with depression, irrespective of their CES-D score. In order to ensure that our results did not depend on the cutpoint used for the CES-D, and to examine the association between incremental increases in depressive symptoms and risk of dementia, we also calculated the risk of dementia for each 10-point increase in the 60-point CES-D. We used Cox proportional hazards regression models to compare participants with and without depression with respect to the development of incident dementia and to estimate the hazard ratio (HR) associated with each 10-point increase in the CES-D. Two main models were examined. Model 1 adjusted for age and sex and model 2 further adjusted for education, homocysteine, and $A P O E \in 4$. In 2 supplemental models that built on model 1 , we also adjusted for major vascular risk factors (prevalent stroke and history of diabetes, cardiovascular disease, and hypertension) and for current smoking, alcohol use, and waist-hip ratio. We confirmed that the assumption of proportionality of hazards was met by including terms for interactions with follow-up time. The risk of dementia is more likely to change as a function of age than of calendar time; therefore, in our primary analyses, we used age as the time scale, adjusting for left truncation at entry. Survival age was defined as age at diagnosis of dementia in persons who developed dementia or age at death if the person died 
Table 1 Baseline characteristics of participants according to CES-D depression status $^{a}$

\begin{tabular}{|c|c|c|c|}
\hline & \multicolumn{2}{|c|}{ Depressive symptoms } & \multirow[b]{2}{*}{$p$} \\
\hline & No $(n=824)$ & Yes $(n=125)$ & \\
\hline Age, y, mean \pm SD & $79 \pm 5$ & $81 \pm 6$ & 0.017 \\
\hline Women & 62 & 75 & 0.004 \\
\hline \multicolumn{4}{|l|}{ Education } \\
\hline No HS degree & 3 & 7 & \\
\hline HS degree only & 26 & 40 & \\
\hline Some college & 37 & 33 & \\
\hline College degree & 35 & 20 & $<0.001$ \\
\hline Current smoking & 8.4 & 8.8 & 0.882 \\
\hline Any alcohol use & 50 & 27 & $<0.001$ \\
\hline Median homocysteine & 11.0 & 12.1 & $0.064^{b}$ \\
\hline APOE $\epsilon 4$ & 19 & 23 & 0.391 \\
\hline Prevalent stroke & 5.2 & 5.6 & 0.859 \\
\hline History of diabetes & 12.8 & 19.6 & 0.058 \\
\hline Stage $1+$ hypertension & 72 & 72 & 0.903 \\
\hline History of CVD & 29 & 46 & $<0.001$ \\
\hline Waist-hip ratio, mean \pm SD & $0.93 \pm 0.09$ & $0.92 \pm 0.08$ & 0.278 \\
\hline Antidepressant medications & 3.4 & 8.8 & 0.005 \\
\hline
\end{tabular}

Abbreviations: CES-D = Center for Epidemiologic Studies Depression Scale; CVD = cardiovascular disease; $\mathrm{HS}=$ high school.

${ }^{\text {a }}$ All values are percentages unless otherwise noted.

b Tested using log. thus, we did not perform the analyses separately in men and women.

RESULTS In this sample of 949 participants, the mean age was 79 years (SD 5) and 63.6\% were women. The mean CES-D score of the sample at baseline was 7.5 (SD 7.8) with 125 (13.2\%) participants having a score of 16 or higher and being classified as depressed; an additional 39 (4.1\%) were depressed based on medication records. Baseline characteristics of participants according to depression status are presented in table 1. Depressed subjects were significantly older, were more likely to be female, and were less likely to have education beyond high school than patients who were not depressed. Patients who were depressed were less likely to drink alcohol and more likely to have a history of cardiovascular disease. Homocysteine level, waist-hip ratio, current smoking status, and history of stroke, diabetes, or hypertension did not vary by depression status.

During the 17-year follow-up period, dementia developed in 164 subjects. After adjusting for demographic characteristics, homocysteine, and APOE $\epsilon 4$, depressed participants (CES-D $\geq 16$ ) were more than 1.5 times as likely to develop dementia (HR $1.72,95 \%$ confidence interval [CI] $1.04-2.84, p=$ $0.035)$ than those who were not depressed (CES-D $<16$ ) (table 2, dementia model 2). Similar results were observed when we included antidepressant medications in the definition of depression; depressed participants were significantly more likely to develop dementia (HR 1.67, 95\% CI 1.05-2.66, $p=0.031)$. We also examined the impact of each 10 -point increase in depressive symptoms, using total CES-D score, on dementia risk and found a nearly $50 \%$ increased risk of incident dementia (HR free of dementia; the remaining subjects were censored at the last age at which they had been verified to be free of dementia.

Based on previous findings that have reported effect modification for the association between depression and dementia by sex, ${ }^{13,14} A P O E \epsilon 4,{ }^{25}$ and education, ${ }^{12}$ we also tested whether there were significant interactions between depression and these $3 \mathrm{fac}-$ tors. We found a significant interaction between depression and sex for the risk of dementia, but the interaction was dependent on the definition of depression (CES-D score only, $p=0.063$, and CES-D score + antidepressant medication use, $p=0.033$ );

Table 2 Association between depressive symptoms and the risk of dementia and Alzheimer disease: The Framingham Heart Study (original cohort)

\begin{tabular}{|c|c|c|c|c|c|c|c|c|c|c|c|c|}
\hline \multirow[b]{3}{*}{ Cases/N } & \multicolumn{6}{|c|}{ Dementia } & \multicolumn{6}{|c|}{ AD only } \\
\hline & \multicolumn{3}{|c|}{ Model $1(164 / 949)^{a}$} & \multicolumn{3}{|c|}{ Model $2(137 / 736)^{b}$} & \multicolumn{3}{|c|}{ Model $1(136 / 949)^{a}$} & \multicolumn{3}{|c|}{ Model $2(115 / 736)^{b}$} \\
\hline & HR & $95 \% \mathrm{Cl}$ & $p$ & HR & $95 \% \mathrm{Cl}$ & $p$ & HR & $95 \% \mathrm{Cl}$ & $p$ & HR & $95 \% \mathrm{Cl}$ & $p$ \\
\hline CES-D $\geq 16$ & 1.78 & 1.17-2.71 & 0.007 & 1.72 & $1.04-2.84$ & 0.035 & 1.89 & $1.21-2.96$ & 0.005 & 1.76 & 1.03-3.01 & 0.039 \\
\hline $\begin{array}{l}\text { CES-D } \geq 16 \text { or } \\
\text { antidepressant } \\
\text { prescription }^{c}\end{array}$ & 1.74 & $1.17-2.59$ & 0.006 & 1.67 & $1.05-2.66$ & 0.031 & 1.65 & $1.06-2.56$ & 0.026 & 1.57 & $0.94-2.62$ & 0.082 \\
\hline $\begin{array}{l}\text { Continuous } \\
\text { CES-D } D^{d}\end{array}$ & 1.39 & 1.16-1.65 & $<0.001$ & 1.46 & 1.18-1.79 & $<0.001$ & 1.35 & $1.11-1.63$ & 0.003 & 1.39 & $1.11-1.75$ & 0.005 \\
\hline
\end{tabular}

Abbreviations: AD = Alzheimer disease; CES-D = Center for Epidemiologic Studies Depression Scale; CI = confidence interval; $\mathrm{HR}=$ hazard ratio.

a Model 1 adjusted for age and sex.

b Model 2 adjusted for age, sex, education, homocysteine, and APOE $\epsilon 4$.

c Definition of depression based on CES-D score $\geq 16$ or use of antidepressant medications.

${ }^{d}$ CES-D score examined in 10-point increments. 
Table 3 Association between depressive symptoms and the risk of dementia and Alzheimer disease among participants without possible cognitive impairment at baseline: The Framingham Heart Study

\begin{tabular}{|c|c|c|c|c|c|c|c|c|c|c|c|c|}
\hline \multirow[b]{3}{*}{ Cases/N } & \multicolumn{6}{|c|}{ Dementia } & \multicolumn{6}{|c|}{$A D$ only } \\
\hline & \multicolumn{3}{|c|}{ Model $1(106 / 882)^{a}$} & \multicolumn{3}{|c|}{ Model $2(89 / 680)^{b}$} & \multicolumn{3}{|c|}{ Model $1(88 / 882)^{a}$} & \multicolumn{3}{|c|}{ Model $2(73 / 680)^{b}$} \\
\hline & HR & $95 \% \mathrm{Cl}$ & $p$ & HR & $95 \% \mathrm{Cl}$ & $p$ & HR & $95 \% \mathrm{Cl}$ & $p$ & HR & $95 \% \mathrm{Cl}$ & $p$ \\
\hline CES-D $\geq 16$ & 2.03 & $1.23-3.37$ & 0.006 & 2.10 & $1.16-3.81$ & 0.014 & 2.11 & $1.21-3.65$ & 0.008 & 2.18 & $1.14-4.16$ & 0.019 \\
\hline $\begin{array}{l}\text { CES-D } \geq 16 \text { or } \\
\text { antidepressant } \\
\text { prescription }^{c}\end{array}$ & 1.87 & $1.14-3.05$ & 0.013 & 1.95 & $1.11-3.43$ & 0.020 & 1.85 & $1.08-3.19$ & 0.026 & 1.91 & $1.02-3.57$ & 0.042 \\
\hline $\begin{array}{l}\text { Continuous } \\
\text { CES-D }\end{array}$ & 1.41 & $1.14-1.75$ & 0.002 & 1.50 & $1.16-1.93$ & 0.002 & 1.35 & $1.06-1.72$ & 0.016 & 1.41 & $1.05-1.88$ & 0.022 \\
\hline
\end{tabular}

Abbreviations: $A D=$ Alzheimer disease; $C E S-D=$ Center for Epidemiologic Studies Depression Scale; $\mathrm{Cl}=$ confidence interval; $\mathrm{HR}=$ hazard ratio.

a Model 1 adjusted for age and sex.

${ }^{b}$ Model 2 adjusted for age, sex, education, homocysteine, and APOE $\epsilon 4$.

${ }^{\mathrm{c}}$ Definition of depression based on CES-D score $\geq 16$ or use of antidepressant medications.

d CES-D score examined in 10-point increments.

$1.46,95 \%$ CI $1.18-1.79, p<0.001)$ with each 10 point increase on the 60-point CES-D.

Depression was also associated with an increased risk for $\mathrm{AD}$, which developed in 136 participants. Specifically, depressed participants were more than 1.5 times as likely to develop $\mathrm{AD}$ compared to those who were not depressed (HR 1.76, 95\% CI 1.03$3.01, p=0.039$; table 2 , AD model 2) and for each 10 -point increase in CES-D score there was a $40 \%$ increased risk of $\mathrm{AD}$ (HR 1.39, 95\% CI 1.11-1.75, $p=0.005)$. When we included use of antidepressant medications in the definition of depression, depressed participants had more than a 50\% increased risk of developing $\mathrm{AD}$ compared to those who were not depressed, although the association did not reach statistical significance (HR 1.57, 95\% CI 0.94-2.62, $p=0.082$ ).

Results were similar when, in 2 supplemental models building on model 1 , which adjusted for age and sex, we additionally adjusted for major vascular risk factors (prevalent stroke and history of diabetes, hypertension, and CVD) and for current smoking, alcohol use, and waist-hip ratio. In the model adjusted for vascular risk factors, depressed participants had a doubled risk for dementia (HR 2.01, 95\% CI $1.20-3.31, p=0.006$; data not shown) and AD (HR 1.97, 95\% CI 1.15-3.39, $p=0.014$ ) compared to those who were not depressed. Similarly, when we adjusted for current smoking status, alcohol use, and waist-hip ratio, depressed subjects had a $80 \%$ increased risk of dementia (HR 1.82, 95\% CI 1.14$2.89, p=0.012)$ and $\mathrm{AD}$ (HR 1.83, 95\% CI 1.11$3.02, p=0.017)$ compared to those who were not depressed.

In order to account for the potential influence of depressive symptoms in the prodromal stage of dementia and to examine reverse causality, among the
949 subjects who did not have dementia, we further excluded 67 participants with possible mild cognitive impairment (MCI). Among the 882 participants without possible MCI or dementia at baseline, a total of 106 participants (72 women, 34 men) developed dementia and 88 developed $\mathrm{AD}$ ( 57 women, 31 men). In this more conservatively defined subsample, similar results were observed. Participants who were depressed, defined as CES-D score $\geq 16$ as well as defined as CES-D score $\geq 16$ or use of antidepressant medications, were twice as likely to develop dementia (HR 2.10, $95 \%$ CI $1.16-3.81, p=0.014$, and HR $1.95,95 \%$ CI $1.11-3.43, p=0.020$, table 3, dementia model 2) and $\mathrm{AD}$ (HR 2.18, 95\% CI 1.14-4.16, $p=0.019$, and HR 1.91, 95\% CI 1.02-3.57, $p=0.042$, table $3, \mathrm{AD}$ model 2) after adjusting for demographic factors, homocysteine, and $A P O E \epsilon 4$, compared to participants who were not depressed. For each 10-point increase in CES-D score, there was a $50 \%$ increased risk of dementia (HR 1.50,95\% CI 1.16-1.93, $p=0.002$ ) and a $40 \%$ increased risk for AD (HR 1.41, 95\% CI 1.05$1.88, p=0.022)$. Results were similar when we excluded participants who developed dementia within 3 years of the depression measurement $(\mathrm{n}=104$; data not shown).

DISCUSSION In a population-based cohort study, depression was associated with a significantly increased risk of incident dementia and $\mathrm{AD}$ in older men and women over a 17-year follow-up period. Being depressed nearly doubled the risk of dementia and $\mathrm{AD}$ after controlling for age and sex. Results were unchanged when we controlled for education, homocysteine, and $A P O E \epsilon 4$ and when persons with possible MCI were excluded.

Depression, characterized by a history of major depression or high depressive symptomatology, has 
been identified as a risk factor for both dementia and cognitive decline in a number of previously published studies. ${ }^{1-7}$ Inconsistency in results, however, has been reported, particularly from prospective studies. ${ }^{9-11}$ In addition, some studies have reported significant differences in dementia risk only for specific subgroups related to education ${ }^{12}$ or sex. ${ }^{13,26}$ One limitation of previously published prospective studies of the association between depression and dementia risk is relatively short follow-up periods following assessment of depression. With few exceptions, ${ }^{6}$ studies report $\leq 5$ years of follow-up between the assessment of depression and diagnosis of dementia., 7,9,11,12 With short follow-up times between the assessment of depression and dementia diagnosis, it is difficult to determine whether depression increases the risk for dementia or depressive symptomatology is increased as a result of the dementing process. Our findings support previous work identifying depression as a risk factor for dementia and $\mathrm{AD}$, and extend this finding to a follow-up period of up to 17 years.

It is unclear whether depression is a risk factor for dementia or whether depressive symptoms are an early sign of dementia pathology. ${ }^{8,9,12}$ Causality is difficult to resolve and the methods by which it is examined vary among previously published studies on the association between late-life depression and the risk of dementia. A number of studies have excluded subjects with MCI, based on MMSE scores, to account for depression as a prodromal sign of dementia. ${ }^{12}$ Other studies address causality through statistical modeling (e.g., controlling for baseline cognitive performance). ${ }^{6}$ Due to the careful case ascertainment and defining of the date of dementia onset, we did not exclude participants based on MMSE scores from our main analyses. However, results were consistent when we excluded the subgroup of participants with possible MCI, addressing the issue of reverse causality and suggesting that depression in these participants was not associated with early clinical dementia.

There are a number of mechanisms by which depression may impact the risk of dementia. Chronic inflammatory changes which occur in depression, potentially as a result of stress, may contribute to the development of dementia and AD. ${ }^{27}$ In particular, interleukin-6, C-reactive protein, and tumor necrosis factor $-\alpha$ are increased in persons with depression and may also be associated with an increased risk for dementia. ${ }^{28,29}$ Brain-derived neurotrophic factor, a neurotrophin that may be involved in synaptic plasticity, is decreased in depression, and low levels of brain-derived neurotrophic factor are also associated with dementia, particularly AD. ${ }^{30,31}$

Depression may also be associated with vascular factors as described by the vascular depression hypothesis, which postulates that vascular pathology contributes to the pathogenesis of depression in late life. ${ }^{32}$ Indeed, cerebrovascular disease, observed in neuroimaging studies and controlled for white matter lesion severity, ${ }^{33}$ and increased arterial stiffness, assessed using carotid artery distensibility and pulse wave velocity, ${ }^{34}$ have been observed in patients with depressive symptoms in large epidemiologic studies. However, controlling for major vascular risk factors did not change our results.

Several lifestyle factors associated with longstanding depression, such as diet, physical activity, and social engagement, may increase the risk for dementia. Population-based epidemiologic studies have shown that vitamin B12 deficiency and high levels of homocysteine are associated with depression in older adults and with an increased risk for dementia and AD. ${ }^{35,36}$ A bidirectional association between physical activity and depression has been suggested, with some finding that physical activity declines as a result of depression ${ }^{37}$ and others suggesting that change in activity is a potential risk factor for depression. ${ }^{38}$ Change in physical activity may also be a marker of depression. Depressed persons may decrease their levels of social engagement, which is also associated with an increased risk for dementia. ${ }^{39}$

This study has a number of strengths, including a population-based cohort design with up to 17 years of follow-up for incident dementia and rigorous dementia case ascertainment methods. The cohort is well-characterized with respect to cognitive and health factors; thus, we were able to control for a number of potentially confounding factors. The limitations of this study include the lack of inclusion of diverse ethnic/racial groups and of psychiatric documentation of the presence or absence of depression. We were unable to examine the duration of depressive symptoms and did not have information on adherence to treatment with antidepressants. We were also unable to exclude the possibility that depression allows for earlier detection of dementia and we could not control for white matter changes since there was no systematic MRI measurement of the cohort at the time depression was assessed. In addition, we could not control for physical activity, social engagement, or sleep patterns, which are associated with the risk for dementia and may be altered in depression. As with all longitudinal studies, the follow-up population reported here is younger and healthier than the baseline original Framingham Heart Study cohort.

\section{AUTHOR CONTRIBUTIONS}

Statistical analysis was conducted by Dr. Alexa Beiser.

\section{STUDY FUNDING}

Supported by the Framingham Heart Study's National Heart, Lung, and Blood Institute contract (N01-HC-25195) and by grants (AG16495 and 
AG08122) from the National Institute on Aging and a grant (NS17950) from the National Institute of Neurological Disorders and Stroke.

\section{DISCLOSURE}

Dr. Saczynski reports no disclosures. Dr. Beiser receives royalties from the publication of Introductory Applied Statistics (Brooks Cole, 2005) and receives research support from the NIH (NINDS 2 R01NS017950-28 [biostatistician], NIA 5 R01 AG08122 [biostatistician], NIA 2 R01 AG16495 [biostatistician], NINDS 1 R01 AG033040-01 [biostatistician], and NHLBI R01 HL093029-01A1 [biostatistician]). Dr. Seshadri serves as an Associate Editor of the Journal of Alzheimer's Disease and receives research support from the NIH (NIA R01 AG08122 [investigator], NINDS R01 NS017950-28 [investigator], NIA R01 AG16495 [investigator], NIA 1 R01 AG033040-01 [investigator], NIA P30AG013846 [investigator], NINDS R01 NS062877-01 [PI of subcontract], NIA R01 AG033193 [PI], NIA R01 AG031287 [PI], and NHLBI R01HL093029 [PI of subcontract]). Dr. Auerbach has served on a scientific advisory board for and has received speaker honoraria from Forest Laboratories, Inc. and has served on speakers' bureaus for Forest Laboratories, Inc. and Takeda Pharmaceutical Company Limited. Dr. Wolf receives royalties from the publication of the 5th edition of Stroke: Pathophysiology, Diagnosis, and Management (Elsevier, 2008) and receives research support from the NIH (NHLBI N01-HC-25195 [PI], NINDS 2 R01 NS 17950 [PI], NINDS 1-R01-AG033040 [PI], NIA 5-R01-AG-08122 [PI], NIA 1-R01-AG16495 [PI], NHLB R01HL093029-01A1 [Co-I], NIA R01-AG033193 [Co-I], NIA R01-AG029451 [Co-I], NHLBI 1-R01HL083197 [Co-I], NIA R01 NS062877-01 [Co-I], and NIA R01 AG 031287-01A2 [Co-I]). Dr. Au receives research support from the NIH (NIA 5 R01 AG08122 [investigator], NIA 2 R01 AG16495 [investigator], NINDS 2 R01 NS017950 [neuropsychologist], NIA 1 R01 AG033040 [investigator], and NIA R01-AG029451 [neuropsychologist]).

Received December 10, 2009. Accepted in final form March 10, 2010.

\section{REFERENCES}

1. Barnes DE, Alexopoulos GS, Lopez OL, Williamson JD, Yaffe K. Depressive symptoms, vascular disease, and mild cognitive impairment: findings from the Cardiovascular Health Study. Arch Gen Psychiatry 2006;63:273-279.

2. Jorm AF. History of depression as a risk factor for dementia: an updated review. Aust NZ J Psychiatry 2001;35: $776-781$.

3. Jorm AF, van Duijn CM, Chandra V, et al. Psychiatric history and related exposures as risk factors for Alzheimer's disease: a collaborative re-analysis of case-control studies: EURODEM Risk Factors Research Group. Int J Epidemiol 1991;20:S43-S47.

4. Speck CE, Kukull WA, Brenner DE, et al. History of depression as a risk factor for Alzheimer's disease. Epidemiology 1995;6:366-369.

5. Green RC, Cupples LA, Kurz A, et al. Depression as a risk factor for Alzheimer disease: the MIRAGE study. Arch Neurol 2003;60:753-759.

6. Wilson RS, Barnes LL, Mendes de Leon CF, et al. Depressive symptoms, cognitive decline, and risk of $\mathrm{AD}$ in older persons. Neurology 2002;59:364-370.

7. Devanand DP, Sano M, Tang M-X, et al. Depressed mood and the incidence of Alzheimer's disease in the elderly living in the community. Arch Gen Psychiatry 1996;53:175-182.

8. Berger AK, Fratiglioni L, Forsell Y, Winblad B, Backman L. The occurrence of depressive symptoms in the preclinical phase of AD: a population-based study. Neurology 1999;53:1998-2002.

9. Chen P, Ganguli M, Mulsant BH, DeKosky ST. The temporal relationship between depressive symptoms and dementia: a community-based prospective study. Arch Gen Psychiatry 1999;56:261-266.
10. Becker JT, Chang YF, Lopez OL, et al. Depressed mood is not a risk factor for incident dementia in a communitybased cohort. Am J Geriatr Psychiatry 2009;17:653-663.

11. Henderson AS, Korten AE, Jacomb PA, et al. The course of depression in the elderly: a longitudinal communitybased study in Australia. Psychol Med 1997;27:119-129.

12. Geerlings MI, Bouter LM, Schoevers R, et al. Depression and risk of cognitive decline and Alzheimer's disease: results of two prospective community-based studies in the Netherlands. Br J Psychiatry 2000;176:568-575.

13. Fuhrer R, Dufouil C, Dartigues J. Exploring sex differences in the relationship between depressive symptoms and dementia incidence: prospective results from the PAQUID Study. J Am Geriatr Soc 2003;51:1055-1063.

14. Forno G, Palermo M, Donohue J, Karagiozis H, Zonderman A, Kawas C. Depressive symptoms, sex, and risk for Alzheimer's disease. Ann Neurol 2005;57:381-387.

15. Farmer ME, White LR, Kittner SJ, et al. Neuropsychological test performance in Framingham: a descriptive study. Psychol Rep 1987;60:1023-1040.

16. Radloff L. The CES-D Scale: a self-report depression scale for research in the general population. Appl Psychol Methods 1977;1:385-401.

17. Seshadri S, Beiser A, Kelly-Hayes M, et al. The lifetime risk of stroke: estimates from the Framingham Study. Stroke 2006;37:345-350.

18. American Psychiatric Association. Diagnostic and Statistical Manual of Mental Disorders: DSM-IV. Washington, DC: American Psychiatric Association; 1994.

19. Berg L. Clinical Dementia Rating (CDR). Psychopharmacol Bull 1988;24:637-639.

20. McKhann G, Drachman D, Folstein M, Katzman R, Price D, Stadlan EM. Clinical diagnosis of Alzheimer's disease: report of the NINCDS-ADRDA Work Group under the auspices of Department of Health and Human Services Task Force on Alzheimer's Disease. Neurology 1984;34: 939-944

21. Araki A, Sako Y. Determination of free and total homocysteine in human plasma by high-performance liquid chromatography with fluorescence detection. J Chromatogr 1987;422:43-52.

22. Selhub J, Jacques PF, Wilson PWF, Rush D, Rosenberg IH. Vitamin status and intake as primary determinants of homocystinemia in an elderly population. JAMA 1993; 270:2693-2698.

23. Ordovas JM, Litwack-Klein L, Wilson PW, Schaefer MM, Schaefer EJ. Apolipoprotein E isoform phenotyping methodology and population frequency with identification of apoE1 and apoE5 isoforms. J Lipid Res 1987;28:371-380.

24. Welty FK, Lahoz C, Tucker KL, Ordovas JM, Wilson PWF, Schaefer EJ. Frequency of ApoB and ApoE gene mutations as causes of hypobetalipoproteinemia in the Framingham offspring population. Arterioscler Thromb Vasc Biol 1998;18:1745-1751

25. Irie F, Masaki KH, Petrovitch $\mathrm{H}$, et al. Apolipoprotein E $\epsilon 4$ allele genotype and the effect of depressive symptoms on the risk of dementia in men: the Honolulu-Asia Aging Study. Arch Gen Psychiatry 2008;65:906-912.

26. Dal Forno G, Palermo MT, Donohue JE, Karagiozis H, Zonderman AB, Kawas CH. Depressive symptoms, sex, and risk for Alzheimer's disease. Ann Neurol 2005;57:381-387.

27. Leonard BE. Inflammation, depression and dementia: are they connected? Neurochem Res 2007;32:1749-1756. 
28. Engelhart MJ, Geerlings MI, Meijer J, et al. Inflammatory proteins in plasma and the risk of dementia: the Rotterdam Study. Arch Neurol 2004;61:668-672.

29. Miller GE, Stetler CA, Carney RM, Freedland KE, Banks WA. Clinical depression and inflammatory risk markers for coronary heart disease. Am J Cardiol 2002;90:1279-1283.

30. Zuccato C, Cattaneo E. Brain-derived neurotrophic factor in neurodegenerative diseases. Nat Rev Neurol 2009;5:311-322.

31. Tsai S-J. Brain-derived neurotrophic factor: a bridge between major depression and Alzheimer's disease? Med Hypoth 2003;61:110-113.

32. Alexopoulos GS, Meyers BS, Young RC, Campbell S, Silbersweig D, Charlson M. 'Vascular depression' hypothesis. Arch Gen Psychiatry 1997;54:915-922.

33. Steffens DC, Helms MJ, Krishnan KR, Burke GL. Cerebrovascular disease and depression symptoms in the Cardiovascular Health Study. Stroke 1999;30:2159-2166.

34. Tiemeier H, Breteler MM, van Popele NM, Hofman A, Witteman JC. Late-life depression is associated with arterial stiffness: a population-based study. J Am Geriatr Soc 2003;51:1105-1110.
35. Tiemeier H, van Tuijl HR, Hofman A, Meijer J, Kiliaan AJ, Breteler MMB. Vitamin B12, folate, and homocysteine in depression: the Rotterdam Study. Am J Psychiatry 2002;159:2099-2101.

36. Penninx BWJH, Guralnik JM, Ferrucci L, Fried LP, Allen RH, Stabler SP. Vitamin B12 deficiency and depression in physically disabled older women: epidemiologic evidence from the Women's Health and Aging Study. Am J Psychiatry 2000;157:715-721.

37. van Gool CH, Kempen GI, Penninx BW, Deeg DJ, Beekman AT, van Eijk JT. Relationship between changes in depressive symptoms and unhealthy lifestyles in late middle aged and older persons: results from the Longitudinal Aging Study Amsterdam. Age Ageing 2003;32:81-87.

38. Lampinen P, Heikkinen R-L, Ruoppila I. Changes in intensity of physical exercise as predictors of depressive symptoms among older adults: an eight-year follow-up. Prev Med 2000;30:371-380.

39. Saczynski JS, Pfeifer LA, Masaki K, et al. The effect of social engagement on incident dementia: the HonoluluAsia Aging Study. Am J Epidemiol 2006;163:433-440.

\section{Activate Your Online Subsciption}

Go to www.neurology.org and click on "Activate/Manage Your Subscription" (in left-hand column under "Journal Resources."). Then click on the "Activate Your Individual Member or NonMember Subscription" link and follow the online instructions.

\section{The NEW Neurology ${ }^{\circledR}$ online offers:}

- Sign up for Table of Contents and Topic Alerts

- "Personal folders" for articles and searches

- Resident and Fellow subsite

- Links to Neurology Now ${ }^{\circledR}$, Neurology Today ${ }^{\circledR}$, and Continuum $^{\circledR}$

- Mobile device download link

- Detailed podcast descriptions
- RSS Feeds of current issue and podcasts

- AAN web page links

- Social media including Facebook and Twitter links

- Increased search capability

- Enhanced navigation format

- Highlighted articles 